\title{
EL CONCEPTO DE SOSTENIBILIDAD TURÍSTICA EN LOS LIBROS DE INGLÉS APLICADO AL TURISMO
}

\author{
Paloma López-Zurita \\ María Vázquez Amador \\ $M^{a}$ Carmen Lario de Oñate \\ Universidad de Cádiz.
}

\section{RESUMEN}

2017 fue designado como el Año Internacional del Turismo Sostenible para el Desarro1lo. El concepto de sostenibilidad turística está cada vez más vinculado a las nuevas formas de ocio. Los manuales de Inglés para Turismo deberían asimismo reflejar estas tendencias. El artículo muestra el análisis de contenido de 43 libros de inglés turístico universitario publicados a partir de 1992 para constatar la evolución del enfoque sobre sostenibilidad turística hasta convertirse en un tema habitual en la mayoría de los ejemplares actuales, así como los cambios en la terminología empleada.

Palabras clave: Sostenibilidad; Inglés aplicado al Turismo; Inglés para Fines Específicos (IFE); Didáctica; Manuales.

\section{The concept of sustainable tourism in English for Tourism course books}

\section{ABSTRACT}

2017 is the International Year of Sustainable Tourism for Development. The current concept of tourism sustainability is increasingly linked to new trends of leisure. Course books of English for Tourism, likewise, should reflect these social changes. This article shows the content analysis of 43 textbooks used in teaching English for Tourism, published from 1992. Results verify the evolution of contents related to tourism sustainability, to become a common topic in most current course books, as well as the changes undergone in the terminology used.

Keywords: Sustainability; English applied to Tourism; English for Specific Purposes (ESP); Teaching; Course books.

Fecha de recepción: 16 de febrero de 2017.

Fecha de aceptación: 19 de octubre de 2017.

Departamento de Filología Francesa e Inglesa. Facultad de Ciencias Sociales y de la Comunicación. Avda. de la Universidad, 4. 11405, Jerez de la Frontera. CÁDIZ (España).E-mail: paloma.lopez@uca.es, maria.vazquez@ uca.es, carmen.lario@uca.es 


\section{INTRODUCCIÓN}

Como todos los sectores relacionados con la actividad humana, el sector turístico, desde sus inicios, ha ido adaptándose a los nuevos estilos de vida de la sociedad. Uno de los mayores cambios sufridos tanto por el sector como por el propio turista en los últimos años es la concienciación sobre la influencia que pueden tener en el entorno en el que actúan y sobre las posibles consecuencias negativas que su comportamiento puede acarrear, es decir, sobre la sostenibilidad del turismo. Esta tendencia se traslada a los manuales de estudios turísticos de diferentes disciplinas y, como no puede ser de otra manera, debería trasladarse a los libros de texto de Inglés aplicado al Turismo, que, de igual forma, tienen que ser espejo de esos cambios en la sociedad. Consecuentemente, el objetivo de este estudio es comprobar la inclusión y el tratamiento de contenidos sobre sostenibilidad en dichos libros de texto, así como la evolución conceptual a lo largo de los años como reflejo de estos nuevos escenarios. Para ello, se ha realizado un análisis pormenorizado de una selección de libros utilizados en la enseñanza del inglés turístico en la Universidad, publicados en su mayoría a partir de 1992 (fecha en la que se celebró la Cumbre de la Tierra de Río de Janeiro y donde se acuñó el término 'turismo sostenible'), hasta la actualidad.

Como se desarrolla más adelante, entre los estudios actuales sobre la enseñanza de lenguas y el turismo abundan las publicaciones sobre la relevancia del inglés en el sector, sobre la adecuación de los libros de texto utilizados o sobre la caracterización del inglés turístico. También existen numerosas investigaciones sobre la importancia de la interculturalidad como competencia necesaria para el futuro profesional. Sin embargo, hasta el momento son muy escasos los análisis que aborden el tratamiento de la sostenibilidad en los libros de inglés desde todos los enfoques que la óptica holística de la propia OMT nos señala.

Con la inclusión de temas sobre sostenibilidad turística en nuestros temarios de inglés aplicado damos visibilidad a esta necesaria toma de conciencia a través de la adquisición de estos contenidos y terminología por parte de nuestro alumnado. De igual manera, intentamos colaborar en el desarrollo de una actitud diferente hacia el turismo, tanto desde el punto de vista del turista como desde el punto de vista del profesional de la actividad turística, que contribuirá aún más a expandir este necesario respeto hacia el entorno, su cultura y su medio.

\section{MARCO TEÓRICO}

\subsection{Turismo sostenible}

Hoy en día el turismo se considera una de las industrias más relevantes a nivel mundial, existiendo un flujo constante de viajeros de todo tipo que en los últimos años ha experimentado, pese a la profunda crisis económica internacional, un continuo crecimiento y una profunda diversificación. El flujo de turistas internacionales creció un 3,9\% en 2016 hasta alcanzar los 1.235 millones y las previsiones en enero de 2017 apuntaban a un crecimiento 
de entre el 3\% y el 4\% (OMT, 2017a). Estos flujos turísticos guardan una estrecha relación con el desarrollo de los países configurados como destino, convirtiendo al turismo en un motor clave de su progreso socioeconómico. Entre 2010 y 2030, se prevé que el ritmo de crecimiento de llegadas a estos destinos emergentes $(+4,4 \%$ al año) doble el de llegadas a economías avanzadas (+2,2\% al año) (OMT, 2015).

Tradicionalmente, el turismo se ha entendido como "aquellas actividades que las personas realizan mientras están de viaje en entornos donde no es habitual que se encuentren, cuyos fines son el ocio, los negocios u otros y duran períodos inferiores a un año" (OMT, 1991: 112). Si bien existen multitud de tipos de turismo, dependiendo del interés y objetivo del turista, en las últimas décadas este concepto tradicional se ha ampliado con el del turismo sostenible, adaptándose a distintos entornos económicos, ambientales y sociales (Weaver, 2006; Lane, 2011). Su repercusión es tal que 2017 fue designado como el Año Internacional del Turismo Sostenible para el Desarrollo, la comunidad internacional ha adoptado la nueva Agenda 2030 y la Asamblea General de las Naciones Unidas ha aprobado los nuevos Objetivos de Desarrollo Sostenible (ODS) (OMT, 2017b).

La consolidación progresiva del estado de bienestar tras la Segunda Guerra Mundial produjo un importante crecimiento económico que configuró entre la población al nuevo turista, con más tiempo de ocio, periodos vacacionales obligatorios, reducción de la edad de jubilación, etc. (CCE, 1990: 4). Esta situación de estabilidad produjo un desarrollo desmesurado e incontrolado en algunos sectores, entre ellos el turístico, lo que provocó que en los años 60 se originara una fuerte preocupación por los problemas medioambientales y por el posible agotamiento futuro de los recursos naturales. En este contexto comienzan a celebrarse las llamadas 'Cumbres de la Tierra', Conferencias de Naciones Unidas sobre el Medio Ambiente y el Desarrollo, que comenzaron en Estocolmo en 1972 y que continúan hasta la actualidad. Aunque la toma de conciencia de la importancia del medio ambiente y sus consecuencias para el desarrollo frente al turismo tradicional comienza tímidamente a formarse en esta primera cumbre, la noción de sostenibilidad alcanza cotas mundiales a partir de la celebrada en Río de Janeiro en 1992, donde se acuña oficialmente el concepto 'Desarrollo Sostenible'. Todos estos hitos temporales llevaron a la OMT, partiendo del Informe Brundtland, elaborado por distintas naciones en 1987, a la definición del desarrollo turístico sostenible, siendo aquel que:

Se concibe como una vía hacia la gestión de todos los recursos de forma que puedan satisfacerse las necesidades económicas, sociales y estéticas, respetando al mismo tiempo la integridad cultural, los procesos ecológicos esenciales, la diversidad biológica y los sistemas que sostienen la vida. (OMT, 1999: 22)

Para la OMT (1998: 260) el turismo sostenible se considera un modelo de desarrollo económico diseñado para mejorar la calidad de vida de la población local, proveer de mayor experiencia al visitante, mantener la calidad del medio ambiente, alcanzar los mayores niveles de rentabilidad económica de la actividad turística para los residentes locales y asegurar beneficios por parte de los empresarios turísticos. En una nueva definición más actualizada (OMT, 2012: 1), se resalta este equilibrio y se define el concepto como el "[...] que tiene plenamente en cuenta las repercusiones actuales y futuras, eco- 
nómicas, sociales y medioambientales para satisfacer las necesidades de los visitantes, de la industria, del entorno y de las comunidades anfitrionas". Así, hoy en día para el turista responsable la búsqueda de la sostenibilidad en el viaje parece ser un factor importante a la hora de elegir destino (Pérez, 2004). Por ello, los intentos por integrar la sostenibilidad en la experiencia turística son cada vez mayores, no solo desde el punto de vista medioambiental (Agrusa, Lema, Tanner, Host \& Agrusa, 2010), sino también desde la propia gestión turística (Reynolds, 2013) y la gestión de la calidad a través de procesos en el puesto de trabajo (González, Frías \& Gómez, 2014).

\subsection{El turismo sostenible en los libros de texto de Inglés para Turismo}

El dominio del idioma inglés, como lengua global determinante en el desarrollo del turismo y de la comercialización de sus productos y servicios, es una competencia básica para el avance del futuro profesional del sector y, como tal, debe tener un papel importante en su formación. Son por ello muchos los artículos que hacen referencia a su papel como detonador de la operación turística (Loera \& Escalera, 2014; Vidal, Berlanga de Jesús \& Rumold, 2008) y a la necesidad de su dominio en cualquier tipo de turismo; Pérez (2010) subraya su importancia en el caso del enoturismo, por ejemplo.

Esta necesidad se constata por el aumento de la presencia de temas turísticos en los métodos generalistas de enseñanza de inglés, así como en los dedicados al inglés de los negocios, donde tradicionalmente se incluían, mostrando una clara tendencia hacia su propia identidad individual a través de una mayor producción editorial de manuales de inglés para turismo en las últimas décadas (Varela, 2007).

Entre las investigaciones actuales sobre este tipo de inglés específico, se encuentran, a modo de ejemplo, las siguientes líneas:

- caracterización de sus rasgos principales (Río, 2012);

- clasificación de los géneros discursivos que lo conforman (Calvi, 2014);

- metodologías para la elaboración de materiales didácticos específicos (Pérez, Pupo y Buenaventura, 2011; Moreira, Lopez \& de Abreu, 2015; Bárcena, Martín \& Jordano, 2016)

- evaluación crítica de manuales de enseñanza de inglés en el ámbito turístico para acercarnos más a las mejores estrategias docentes, desde el estudio comparativo de varios métodos (Cabellos \& Castillo, 2002), la especificidad de departamentos concretos dentro del sector (Kuppan, 2008), hasta la implementación de técnicas novedosas, como el storytelling del propio turista (Alcantud, Ricart \& Gregori, 2014) o sus aspectos prágmáticos relacionados con las necesidades del estudiante (Afzali \& Rezapoorian, 2014).

Entre los estudios sobre las competencias necesarias para el profesional del turismo abundan aquellos que destacan el componente intercultural como determinante en el proceso comunicativo en este sector, garante de esa relación bidireccional óptima con la "población local" o las "comunidades anfitrionas" que mencionaba la OMT en sus definiciones anteriores. Este componente cuenta ya con una amplia trayectoria en la enseñanza 
de lenguas, tanto generales (Areizaga, Gómez e Ibarra, 2005) como para fines específicos (Lario \& Vázquez, 2013; Bocanegra, 2015). En el caso del inglés turístico su investigación se implementa desde diferentes enfoques:

- como desarrollo de la competencia intercultural del alumno en su proceso de aprendizaje (Alonso \& González, 2008; Varón, 2009; Cuestas, Zamuner \& Peralta, 2013; Piccioni, 2014; Suau, 2016);

- a través de la evaluación de su presencia en los métodos o manuales existentes (Varela, 2007);

- a través de la creación de nuevos materiales que sí la incluyan (Oliveira, Roberto \& Costa, 2014);

- basándose en las estrategias de enseñanza centradas en el propio profesor para el desarrollo de ese componente comunicativo intercultural (Byram, Gribkova \& Starkey, 2002; Ciolaneau, 2007);

- a través del análisis de los problemas de traducción que estos aspectos culturales conllevan (Soto, 2013), etc.

Podemos, pues, decir que la competencia sobre sostenibilidad ha sido y es ampliamente estudiada en la enseñanza de inglés turístico, pero principalmente desde una de sus múltiples vertientes, la interculturalidad. No hemos constatado ningún estudio específico sobre la incorporación de otros aspectos sobre el turismo sostenible que sí aparecen de hecho en su definición de la OMT, como son los estilos de vida saludables, la repercusión del turismo en la comunidad que lo recibe desde la óptica del trabajador del sector o la inclusión de temas medioambientales. Sin embargo, parece que, aunque todavía tímidamente, el concepto 'sostenible' ya comienza a tenerse en cuenta en la enseñanza de lenguas: Prádanos (2015) lo incluye en las clases de español como lengua extranjera; en el ámbito turístico González (2012) se acerca algo más a este enfoque al realizar su estudio sobre la traducción en el texto de turismo de aventura y Sanmartín (2015) aporta un análisis pragmalingüístico de lo que denomina "lo ecológico y lo sostenible", realizado en normativas y páginas web de promoción turística, sin mencionar su inclusión en los libros de texto especializados en el sector.

Es por ello por lo que hemos abordado este estudio, incluyendo estos parámetros como complemento a los ya existentes.

\section{MARCO METODOLÓGICO}

Para verificar la inclusión de la tendencia actual del turismo hacia un concepto sostenible, hemos llevado a cabo un análisis detallado de los 43 libros (Anexo) utilizados habitualmente por el profesorado para la enseñanza del inglés turístico en la Universidad de Cádiz. El criterio de selección ha sido, por tanto, el de analizar textos cuyos contenidos han sido realmente implementados en el aula durante la larga trayectoria de los estudios turísticos en esta provincia, cuyo origen, a través de diferentes instituciones, se remonta al curso 66-67. Aunque el ejemplar más antiguo data de 1976, la gran mayoría han sido publicados a partir de 1992 hasta la actualidad. 
En la búsqueda de temas que incluyeran el concepto de sostenibilidad en el entorno turístico nos hemos basado en la dimensión ambiental y sociocultural de la sostenibilidad establecida por la propia OMT (2017), que establece cinco parámetros clave: 1 . Crecimiento económico inclusivo y sostenible. 2. Inclusión social, empleo y reducción de la pobreza. 3. Uso eficiente de los recursos, protección ambiental y cambio climático. 4. Valores culturales, diversidad y patrimonio. 5. Comprensión mutua, paz y seguridad.

Adaptando estos parámetros a los contenidos analizados, se han establecido cuatro categorías:

a) En un primer grupo, y de manera gradual desde un enfoque básico hasta un desarrollo completo del concepto 'sostenible', se incluyen aquellos libros que incorporan una visión del turismo enfocado hacia estilos de vida saludables, normalmente dirigido al propio turista, enclavado entre los parámetros 1 y 4 anteriores.

b) Una siguiente categoría engloba aquellos textos que trasladan la sostenibilidad al puesto de trabajo, incluyendo pautas de comportamiento responsable y modelos de seguridad y prevención de riesgos laborales enfocados hacia la calidad del servicio, dirigido en este caso principalmente al profesional del turismo y enclavado en el parámetro 2 anterior.

c) En un tercer grupo se han clasificado los ejemplares que tratan la interculturalidad y los valores de tolerancia y entendimiento entre comunidades lingüísticas iguales o diferentes en contacto directo en el contexto turístico, que engloba los parámetros 4 y 5 .

d) Una cuarta y más amplia agrupación, dado que el tratamiento que se le da en los textos es más profundo, aglutina aquellos manuales en los que se incluye la sostenibilidad desde el punto de vista medioambiental, parámetro 3 de los anteriores.

El análisis del contenido de los ejemplares se ha llevado a cabo de forma manual, a través de la lectura y observación pormenorizada de todos ellos. En el estudio hemos distinguido dos partes que se desarrollan paralelamente: Un análisis cuantitativo en el que se establece el número de ejemplares que responde a las preguntas efectuadas y, a continuación, un análisis cualitativo de las posibles implicaciones que las cifras halladas conllevan. Las cuestiones planteadas son las siguientes: 1. A qué tipología corresponde cada uno de los ejemplares analizados; 2. Cuál es el grado de inclusión de las cuatro categorías anteriores en los mismos; 3 . En esos casos, qué tratamiento se le ha dado, es decir, si se incluye solo a través de actividades puntuales o si incluye unidades específicas sobre el tema, abundando, de nuevo, en los casos sobre interculturalidad y medioambiente, por ser los más habituales; 4. A continuación, y dado que el concepto más desarrollado en estos ejemplares es el relacionado con la sostenibilidad medioambiental, qué términos se usan con más frecuencia en esta categoría para verificar su posible evolución, a través de un vaciado terminológico del discurso utilizado en los libros; 5. Finalmente, se determinan cuáles son los ejemplares que presentan una visión holística de la sostenibilidad turística incluyendo los cuatro enfoques anteriores y a qué niveles de competencia linguiística corresponden. 


\subsection{Tipología de los libros analizados}

La muestra de los 43 libros analizados consta de 22 ejemplares sobre turismo en general, 17 sobre hostelería y restauración, 3 sobre viajes aéreos y 1 sobre oficinas de turismo. Entre los libros sobre turismo general se han incluido tres que, si bien no son específicos para la enseñanza del inglés como lengua extranjera, aportan un contenido en inglés sobre el sector turístico que funciona como un excelente complemento para los alumnos de un nivel avanzado, ya que refuerzan sus conocimientos del idioma y de la industria turística al mismo tiempo. Son los ejemplares 6, 27 y $43^{1}$.

\section{Gráfico 1 \\ TIPOLOGÍA DE LOS LIBROS ANALIZADOS}

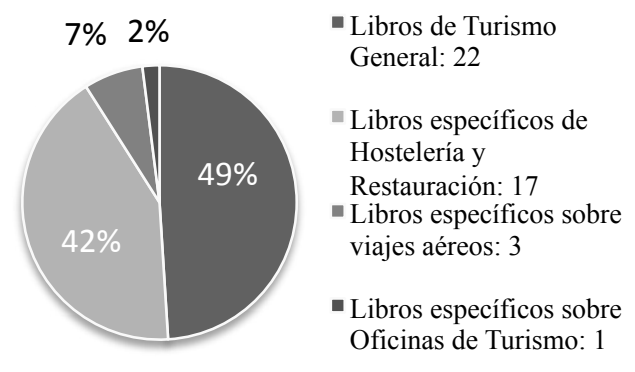

\section{RESULTADOS}

De los 43 libros de texto analizados tan solo 7 no incluyen entre sus contenidos alusión alguna a la sostenibilidad turística. Son ejemplares en su mayoría de nivel inicial. 5 corresponden a la tipología de libros específicos sobre hotelería y restauración $(1,2,4,8$ y 9), 1 sobre viajes aéreos (41) y tanto solo 1 ejemplar sobre turismo general (39). Los 36 restantes abordan el tema como sigue:

\subsection{Sostenibilidad y formas de vida saludables}

El ocio y el tiempo libre han cobrado una dimensión novedosa en los últimos tiempos, convirtiéndose en muchas ocasiones en selectivos e incrementando la preocupación por realizar actividades que repercutan beneficiosamente tanto en el turista como en el entorno en el que se practican. Así, se desarrolla un turismo de salud que incluye desde estancias en circuitos de spa, balnearios, prácticas deportivas, etc., hasta la toma de conciencia de una alimentación saludable, con la repercusión que este modelo tiene en un tipo de turismo inclusivo y sostenible. De manera general, hemos denominado a este apartado 'formas de vida saludables'. En este caso, 25 de los 43 ejemplares analizados (58\%) no incluyen nada específico sobre este tema. Entre ellos, 17 (68\%) corresponden a Hostelería y restauración

1 Las referencias a los 43 ejemplares analizados es muy recurrente en el texto. Por ello, se aludirá a los diferentes manuales a través del número asignado en el Anexo a este trabajo. 
(el $100 \%$ de los analizados), 4 (16\%) a Turismo general y se incluyen también en este apartado los 3 de viajes aéreos (12\%) y el relativo a oficinas de turismo. Sin embargo, sí aparece en 15 de los 21 libros de Turismo general analizados, lo que supone un 71,5\% en este tipo de ejemplares. Se trata, pues, de un tema más relevante para aquellos manuales que enfocan el turismo desde una óptica global, sin una especialidad en concreto:

\section{Gráfico 2 \\ SOSTENIBILIDAD Y FORMAS DE VIDA SALUDABLES}

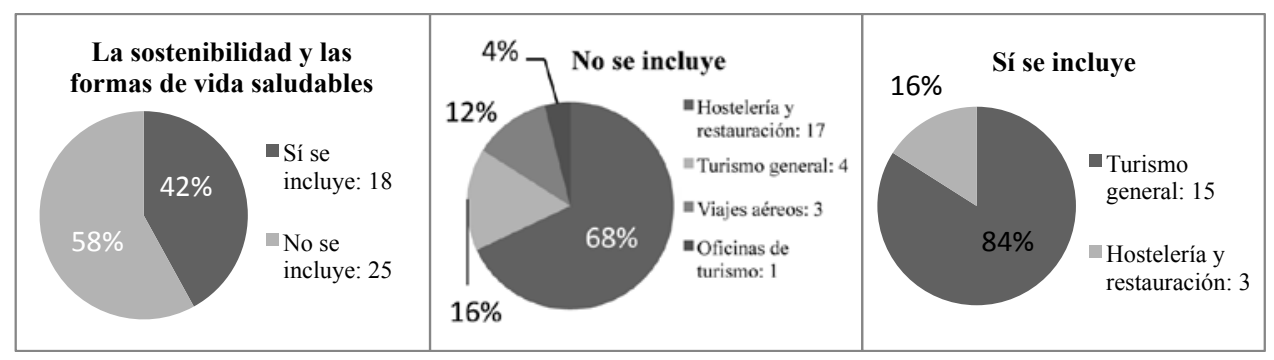

Estos contenidos se desarrollan como actividades diversas del tipo Escape and Enlightment Holidays (33: 91), Food preparation for Healthy Eating (15: 36-56), Eating habits, (26: 19), etc., englobadas en unidades con otros temas como eje central, en 4 de los ejemplares analizados $(5,40,41$ y 42); en los 14 restantes se les dedica toda una unidad. A modo de ejemplo: Holiday Health (34: Unidad 4); Paradise Found (19: Unidad 6); The benefits of holidays for young people. (36: Unidad 11)

En cuanto al nivel de los ejemplares que sí incluyen este parámetro, solo en 1 caso se trata de un nivel elemental, 10 se encuentran en un rango de pre-intermedio a intermedio, 6 corresponden a un nivel intermedio alto y solo 1 tiene un nivel avanzado.

El turismo vinculado a la salud suele aparecer en muchos casos relacionado con la práctica de deportes al aire libre, pero poco a poco se postula como un tipo diferente de turismo emergente. Así, se menciona como tal en 31, que incluye las características de los diferentes tipos de turismo y de motivaciones para el turista e incluso se aporta su definición, diferenciándola claramente del turismo de deporte:

Health Tourism: For people who seek relaxation improving their physical and spiritual well-being: spas, meditation, fitness centers, etc." [...] "Sport Tourism: For people who travel to places where they can practice their favourite sports they might not be able to do in their place of residence: golf, ski, cycling, tennis, surfing, etc. (Varela y Bárcena, 2004: 20)

\subsection{Sostenibilidad laboral}

La seguridad en la actividad laboral y los procesos para la mejora de la calidad en los servicios redundan en la calidad de vida del profesional del turismo, encuadrándose, como ya hemos comentado, el concepto amplio de sostenibilidad enfocado a "Mejorar la calidad de vida de la población local, es decir, de la gente que vive y trabaja en el destino" (Rodríguez, 2013: 57). 
Esta mejora tiene también incidencia directa en la propia seguridad del visitante y en la buena marcha de todo este complejo flujo de procedimientos, por lo que también se incluye en algunos de los libros analizados, fundamentalmente en los ejemplares o unidades dedicados al viaje aéreo, donde, por motivos evidentes, la seguridad de todos alcanza más relevancia que en otro tipo de viajes. Por ello, se incluyen procesos como Airport procedures o cabin crew procedures (25: 61); Security and the aircraft safety features; safety procedures (42: 21); Emergency Procedures (40: Apendix III), que pueden llegar a ser vitales literalmente, tanto para el viajero como para el profesional.

Como se observa en el Gráfico 3 a continuación, de los 43 libros analizados, el $67 \%$ incluyen con mayor o menor profundidad estos temas (29 casos) y el $33 \%$ del total no hace alusión alguna a este concepto de sostenibilidad (14 ejemplares). Entre los primeros, 15 corresponden a libros sobre Turismo general (52\%), 11 se especializan en el ámbito de Hostelería y restauración (38\%), 2 en viajes aéreos (7\%) y también se incluye en el ejemplar sobre oficinas de viajes (3\%). Teniendo en cuenta que la muestra analizada incluía 17 libros sobre Hostelería y restauración, se observa que la seguridad y la higiene en el puesto de trabajo adquieren un papel muy relevante en este contexto, pues su inclusión alcanza casi el $83 \%$ de los ejemplares con esta especialidad.

El 33\% que no incluye ninguna referencia a la sostenibilidad en el puesto de trabajo corresponde a Hostelería y restauración en 6 casos (43\%), 7 a Turismo general (50\%) y a 1 de los ejemplares sobre viajes aéreos.

\section{Gráfico 3 \\ SOSTENIBILIDAD LABORAL}

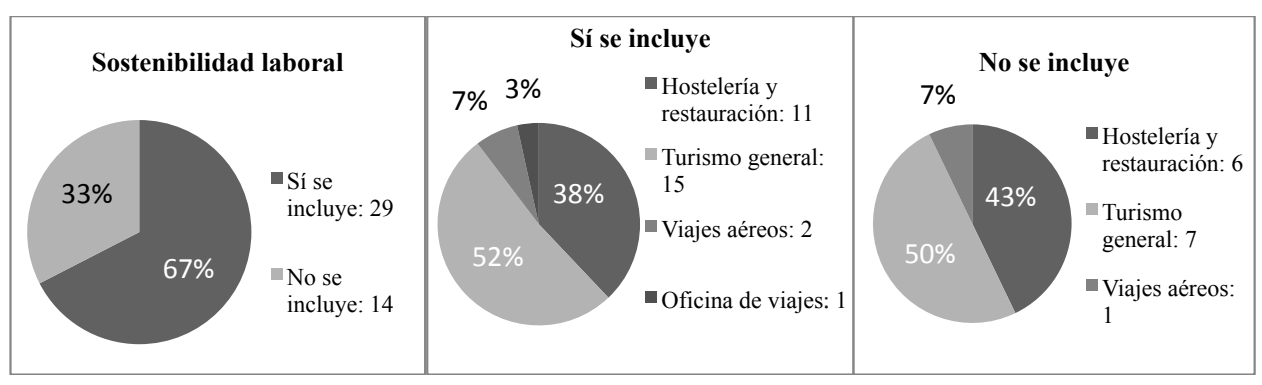

El tratamiento de estos temas también varía. En la mayoría de los casos (19 de los 29 ejemplares), se les dedica una o varias unidades completas, como en 36, por ejemplo, que incluye Book 3: Hotel safety; kitchen safety and sanitation y Part 2: Evacuation; Unit 7: Emergency evaluation Plan. En los 10 restantes su tratamiento se incluye a través de actividades contenidas en el marco de otros temas más generales, como en el caso Travel health and safety (6: 48).

El nivel de los ejemplares es también heterogéneo: elemental en 2 casos, 23 que van desde pre-intermedio a intermedio, 5 de nivel intermedio alto y 1 solo caso de nivel avanzado. 
En cuanto al contenido, se puede distinguir entre aquellos ejemplares que enfocan la seguridad y la calidad hacia el propio trabajador, en busca de una mejora en su situación laboral y aquellos que la focalizan en su repercusión en el usuario de los servicios prestados, o cliente. Entre los primeros se encuentran principalmente los libros sobre Hostelería y restauración. A modo de ejemplo, se citan algunos de los contenidos desarrollados:

Responsibilities: Safety first; Fire procedures. Security measures; Health and Hygiene (5: Secciones 4 y 6);

Hotel safety; Kitchen safety and sanitation, Evacuation; Emergency evaluation Plan; Hotels and Catering (17: Libro 3, Parte 2 y Unidad 7);

Health and Safety at Work (11: Unidad 19);

La salud, la higiene y los conceptos de sanidad (10: Capítulo 5);

Hotel safety; Kitchen safety and sanitation (17: Unidades 7 y 13);

Kitchen organization. Hygiene; Safety (15: 6-8);

Health, safety and security (12: Unidad 23);

En ejemplares de nivel más avanzado se incluyen apartados específicos sobre sostenibilidad laboral medida en términos de calidad como:

Quality standard. Standards of performance (34: Unidad 19);

Employee involvement (35: Unidad 10);

Effective Practices for Hospitality Professionals (13: Appendix 1 and 2);

Safety: Customer Care and Working Procedures, Quality Control (28: Unidad 7);

Quality in Tourism (36: Unidad 6);

Delivering Quality Tourism Services (39: Unidad 3);

The best company to work for (32: Unidad 7), etc., donde se incluyen las mejores prácticas para los trabajadores. Conscientes de su importancia para lograr los estándares deseados en este mismo ejemplar se llega incluso a apuntar: "The image of a hotel depends on the people who work there. Hospitality workers must present themselves as clean, healthy and well-grommed. Here are 20 basic steps towards this goal [...]" (Taylor, 2008: 77).

Entre los ejemplares que también incluyen al turista como usuario de estos servicios, de su seguridad y calidad, aparecen, a modo de ejemplo: Safety Tips (37: Unidad 14); Health and safety abroad (29: 24); Travellers safety tips, safety precautions, hotel security tips (30: Unidad VII); Safety First, Fire Procedures y Security Measures (5: 41, 42 y 43) o Better safe than sorry (26: 92).

\subsection{Sostenibilidad e interculturalidad}

La comunicación intercultural es importante en cualquier contexto pero cobra un papel primordial en el entorno turístico, ya que implica estancias en lugares diferentes al habitual, donde el contacto entre diferentes culturas tiene mayor probabilidad de ocurrir. De ella dependerá en buena medida no solo el bienestar y la confortabilidad del turista sino el propio éxito de la actividad turística. Como reflejo, los ejemplares de inglés turístico analizados prestan bastante importancia a este aspecto: 


\section{Gráfico 4 \\ SOSTENIBILIDAD E INTERCULTURALIDAD}

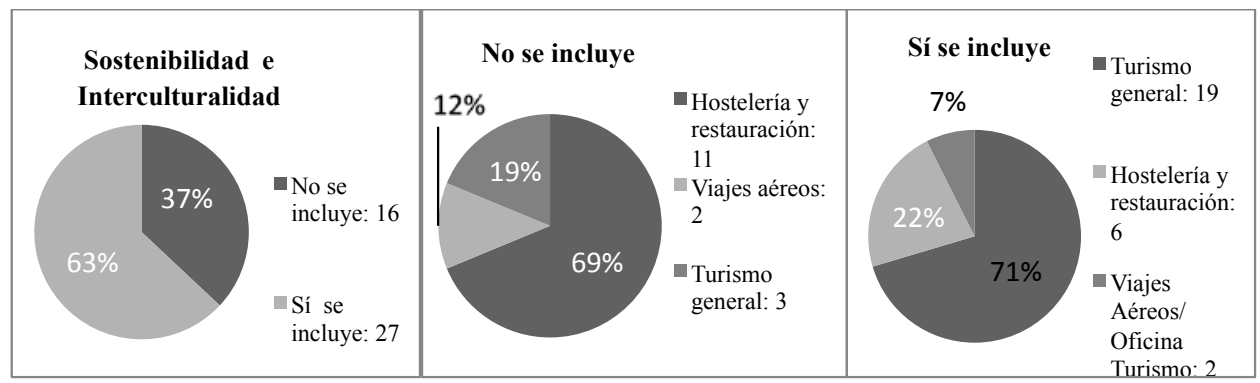

Siguiendo el análisis habitual, en el Gráfico 4 comprobamos que los temas interculturales no se incluyen en 16 de los libros analizados (37\% del total): 2 libros de viajes aéreos (12\%), mucho más específicos y centrados en el tema de la seguridad, como ya se mencionó; 11 ejemplares de hostelería y restauración (69\%) y solo 3 de turismo general (19\%). Por el contrario, los 27 restantes (63\% del total) que sí lo incluyen se distribuyen de la siguiente manera: 6 de hostelería y restauración (22\%), 1 de viajes aéreos, 1 de oficinas de turismo (3,5\% respectivamente) y 19 de turismo general $(71 \%)$, concediendo a los aspectos interculturales la importancia requerida.

El nivel de los ejemplares es elemental en 1 caso, pre-intermedio a intermedio en 18, intermedio alto en 7 y avanzado en 1 .

El tratamiento de la interculturalidad en estos libros de texto es más complejo que en los apartados anteriores, pues suele incluirse con actividades insertas en otros temas pero suelen aparecer en varias unidades, con un tratamiento transversal a las demás cuestiones turísticas, como se observa en los siguientes ejemplos:

El ejemplar 3 no aborda el tema de manera directa. No hay ninguna referencia explícita a la diversidad cultural, pero sí podemos considerar que se incluye de manera tangencial, a través del texto How hoteliers can prepare to welcome their Japanese visitors (p. 105) que, inserto en la Unidad 9 The Business Travel, incide en la importancia de conocer y respetar otras culturas y saber actuar en consecuencia.

La situación que encontramos en el ejemplar 19 es totalmente la inversa. En un primer análisis puede parecer que incluye este tema en las actividades denominadas Social Tourism, o Tourism for all. Sin embargo, al analizar el contenido de las mismas se comprueba que no corresponden a cuestiones interculturales, sino a las instalaciones necesarias para personas discapacitadas, en el primer caso, y al turismo de congresos en el segundo, sin alusión ninguna al asunto analizado en este apartado.

El ejemplar 20 incluye también la problemática del impacto del turismo en las comunidades receptoras en The visitor, the place and the community (p. 38) o The host community (p. 39), aunque está más enfocado hacia la sostenibilidad medioambiental, como se analizará en el siguiente apartado. 
En el caso del ejemplar 22 y su posterior edición como 39, ambos dedican tres unidades completas a la interculturalidad desde diferentes enfoques: Economic and Political Impacts of Tourism (Unidad 11); Environmental and Social/Cultural Impacts of Tourism (Unidad 12) y Sustaining Tourism Benefits (Unidad 13).

El ejemplar 25 abunda en las causas que provocan las corrientes turísticas desde la Unidad 1, donde la actividad The geography of Tourism aborda las diferentes inquietudes que pueden mover al turista, entre ellas la cultura, la historia o la religión de un país. Este ejemplar implementa otras actividades que tratan la sostenibilidad intercultural de manera transversal, entre ellas el texto My Hols. The people of a country makes the difference ( $\mathrm{p}$. 41); International Etiquette (p. 83) y, de nuevo, el impacto negativo sobre las culturas autóctonas en Town seeks refuge from tourist boom. (p. 135).

El ejemplar 26 es otro ejemplo de cómo la interculturalidad se aborda en pequeñas actividades de diferentes unidades. En la página 88, en un contexto distinto, lo aborda a través de la actividad Real Life: Are you politically correct? Las Unidades 1 a 4 se engloban bajo el epígrafe Different kinds of people, haciendo alusión directa al contacto entre culturas con textos como When in Rome ..., por ejemplo. Se enfoca desde diferentes perspectivas, desde la comida, Eating habits, hasta la importancia de la historia y el folclore, History and Folclore (Unidad 48), pasando por las costumbres, como la de dar o no propina, en Is service included? (Unidad 34).

El caso 31 incluye la Unidad 9, en la que se analiza el impacto del turismo desde su repercusión cultural a través del texto Impact of tourism on indigenous people and culture. Este ejemplar, que, como hemos visto anteriormente, incluye definiciones de distintos tipos de turismo, nos aporta también la del denominado 'turismo cultural' en la que, además del propio interés del turista en las atracciones materiales del país a visitar, alude al respeto hacia la cultura y tradiciones autóctonas con las que se desea interactuar, en el texto Cultural tourism: For people who are interested in visiting historical landmarks and monuments as well as enjoying different aspects of the local way of life (Unidad 1).

En el ejemplar 33 se dedica al contacto intercultural buena parte de la Unidad 2, con una práctica oral denominada Different destinations, different customs y el texto What's Tourism?, en el que se recoge el amplio significado que la OMT imprime al concepto 'turismo sostenible', haciendo hincapié en el impacto que el turismo puede provocar en las culturas locales al entrar en contacto con las mismas: "[...] Tourism can save cultures and local way of life, but it can also destroy them. Tourism can change countries -and people- for the better, but it can also change them for the worse". (Walker y Harding, 2011a: 10) Sustancialmente, todo el volumen recoge el mismo espíritu de toma de conciencia y respeto al otro, aunque no sea con unidades completas sino a través de actividades específicas, como acabamos de ver. Se alude al mismo concepto en otros ejercicios de práctica oral, como Tourist motivation (p. 33), Tourist types and holidays types (p. 92), o de lectura, como Cultural differences (p. 89), inserto en la Unidad 10 Holidays with a difference.

Un caso similar lo encontramos en el ejemplar 34, donde el tema intercultural se trata en diferentes actividades transversales a lo largo de todo el libro: Cultural differences in greetings (Unidad 2), Tips on tipping (Unidad 4), Different cultures, different responses (Unidad 7), Cross-cultural Misunderstandings (Unidad 10) o Cultural Awareness, Culture Guide y A Guide to culture and language for the business traveller (Unidad 11), entre otras. 
En 42, aunque se incluye el texto Different types of passengers (p. 21) con un enfoque que abarca posibles grupos diferentes de turistas, de acuerdo a la edad, el estado, la forma de viajar, etc., curiosamente no tiene en cuenta las diferencias culturales como identificador de los mismos.

Para completar los numerosos ejemplos analizados, en el manual 36 el tema vuelve a tratarse de manera transversal en The current state of tourism (Unidad 1), How to behave in meetings (Unidad 7), o Teamwork. (Unidad 9).

\subsection{Sostenibilidad medioambiental}

Definitivamente, si hay un término en estos textos más comúnmente ligado al concepto sostenibilidad es 'medioambiente'. De hecho, su deterioro progresivo fue el detonante para la acuñación del primero. Quizás por ello, el medioambiental es el enfoque sostenible más desarrollado en los libros analizados, incorporando incluso una terminología algo más definida y consistente, donde conceptos como la ecología, el turismo rural o verde, y el propio término 'turismo sostenible' son tratados más ampliamente, abundando en el conocimiento de estos temas y en sus importantes repercusiones. Por estas razones, el análisis de este apartado se configura de manera algo diferente a los anteriores.

El tema medioambiental se incluye en todos los libros de turismo general analizados salvo en dos de nivel inicial, 19 y 30 . No aparece en ninguno de los libros sobre viajes aéreos, oficina de turismo y hostelería y restauración (60\%). Sin embargo, en los 20 casos en los que sí aparece (que suponen un 90,5\% de los 22 libros sobre turismo general analizados) es estudiado mucho más profundamente y con más detalle que los otros tres apartados anteriores.

\section{Gráfico 5}

\section{SOSTENIBILIDAD MEDIOAMBIENTAL}

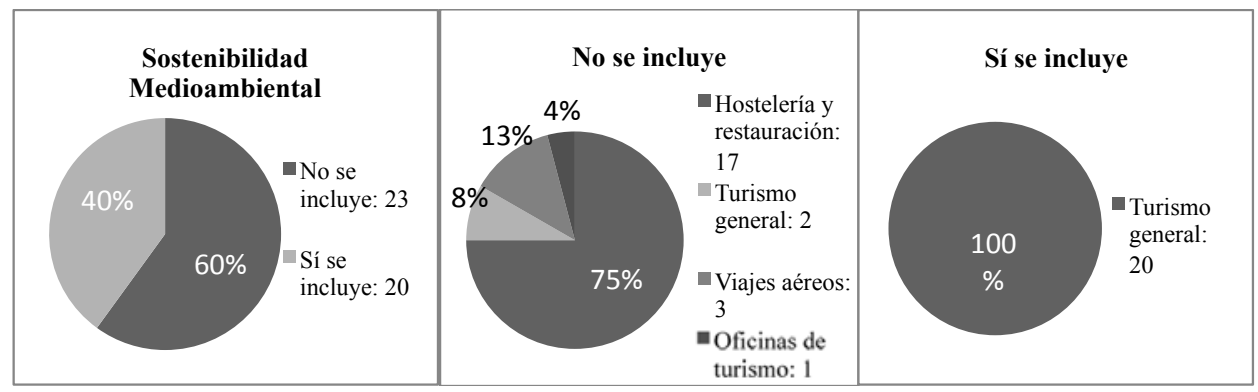

Las alusiones a la sostenibilidad medioambiental se realizan habitualmente de manera explícita, con una terminología más específica que en los campos anteriores. Aunque en muchas ocasiones se aborda a través de unidades completas, como en 18 , $23,24,25,35,38$, etc. la toma de conciencia sobre las repercusiones del turismo en el medio ambiente suele ser también un tema transversal que se incluye en los manuales 
a través de actividades en varias y distintas ocasiones, incluyendo vocablos específicos como green, eco-, sustainable, environmental, etc.; sin embargo, a veces el enfoque se realiza a través de temas que aun sin mencionar estos términos los aluden directamente, como Will our planet survive?(32).

La evolución en la adquisición y fijación del concepto de sostenibilidad en la sociedad y, por lo tanto, en su lengua, se observa en la inclusión y el aumento de una terminología específica para ello: En el libro más antiguo analizado sobre turismo general (19), de 1992, este concepto parece esbozarse tímidamente en algunas unidades, como la 9 Tourism for all o la 10 Social Tourism. Este enfoque, aunque novedoso por entonces, es aún inicial y no muestra una clara definición del concepto, pues, como ya mencionamos anteriormente, al contrario de lo que el propio título de las unidades nos puede llevar a deducir, su contenido no encaja con el concepto de sostenibilidad actual, aunque incluya 'social'. Este vocablo es usado con un matiz hipónimo, ya que carece de las connotaciones que su hiperónimo 'social' tiene en la actualidad, que incluye el respeto hacia las comunidades (en nuestro caso, las receptoras del turismo), hacia la inclusión de grupos y hacia las diferencias culturales y su tolerancia. Su significado en 1992 queda aún inmerso en los tratados internacionales, sin reflejo en los libros de texto. Veamos algunos de los ejemplos detectados:

En la edición de 1994 del ejemplar 20 aparece una terminología más actual, incluyendo aspectos mucho más específicos como Special Interest Holidays (Unidad 1); Rural Tourism (Unidad 2); Tourism and the Environment (Unidad 3), Go on Green (Unidad 4) o National Parks (Unidad 9). Comienza ya en esa década la distinción terminológica entre los tipos de turismo englobados bajo esta idea de sostenibilidad medioambiental. Así, en la Unidad 2 de este mismo manual se incorporan las definiciones de turismo rural y turismo verde:

Rural Tourism - A trip to, or overnight stay in a country area which is neither agricultural or natural and has a low density of population. [...] Green Tourism -The phenomenon of people away from their usual habitat in pursuit of leisure activities in the countryside, excluding such areas as the urbanised coast and ski resorts. (Mioduszewska et al, 1994: 20)

Como se observa, en este caso se trata de definiciones únicamente geográficas, sin el matiz de protección y respeto por la naturaleza. Sin embargo, en el resto de la unidad este aspecto sí aparece implícito en algunas actividades posteriores, como en Nature Conservation (20: 26). Asimismo, el efecto negativo del turismo sobre el medioambiente se aborda con profundidad en la Unidad 3, donde la terminología es mucho más concisa y ya aparecen términos concretos como Environmental Protection o Green Tourism. Green, así como rural, más adelante, ya adquieren connotaciones claras de conciencia medioambiental: "A "Green Tourism Handbook" [...] will encourage good practice in development, management and promotion of tourism, particularly in the countryside". (Mioduszewska et al, 1994: 31) El enfoque de este libro hacia el turismo sostenible es gradual. De una terminología más laxa al inicio se deriva a una más concreta y definida, como se observa en los anteriores ejemplos; en la segunda parte de la Unidad 3 se identifican los problemas causados por el impacto del turismo para, en la Unidad 4, aludir directamente al término sustainable tourism de forma más científica y holística, abarcando todos los aspectos de la sostenibilidad analizados en este artículo: 
Sustainable tourism is not just about valuing landscape and being environmentally friendly. It is also about helping communities by encouraging local employment and the purchase of local products ensures that the long term advantages of tourism are brought to communities in the best possible way. (Mioduszewska et al, 1994: 47)

En el caso 21 se incluye el tema 21, dedicado al vocabulario sobre Environmental and Conservation Issues donde, a través de ejercicios de escritura se utilizan los términos ecotourism, environmentally sensitive, Green globe, responsible, special protection y sustainable, entre otros.

Los ejemplares 22 y 39 incluyen toda la tercera parte completamente dedicada al tema medioambiental, en las unidades 12 y 13: Environmental and Social/Cultural Impacts of Tourism y Sustaining Tourism's Benefits.

La importancia del medio ambiente queda también patente en el ejemplar 24, donde se incluye la unidad 8 Responsible Tourism, con términos como Safe Tourism, abordando directamente las políticas de protección medioambiental y sus implicaciones económicas y ecológicas.

En el caso 25 la Unidad 2 incluye el posible impacto negativo del turismo, a nivel medioambiental y también económico y social:

Although it may help to boost a country's economy, the international tourism industry is fragile. For most people, foreign holidays are a luxury, and when a country is in recession some people will save money by cutting out holidays. This can be economically disastrous for countries which rely heavily on income from tourism. In other parts of the world, from Central America to Western Europe, the development of tourism has also caused widespread environmental damage. (Harding, 2001: 26)

También se dedica la Unidad 12 a los efectos del turismo, con una visión bastante crítica y con muchas actividades para suscitar el debate entre los alumnos. Llega a incluir afirmaciones como "Tourism ultimately spoils a country" (p. 164). Mantiene una concepción de la sostenibilidad bastante amplia, no solo desde el punto de vista medioambiental Green Earth Travel (p. 172), sino también desde el papel del turista How to be a good tourist (p. 170) o del profesional del turismo Responsibility in the tourism industry (p. 172). Incluye asimismo la Sección 3 Tourism and the Environment, donde se proponen para discusión los 10 principios del turismo sostenible, haciéndose eco de lo que ya se venía debatiendo ampliamente en los foros internacionales:

Using resources sustainably. Reducing over-consumption and waste. Maintaining diversity. Integrating tourism into planning. Supporting local economies. Involving local communities. Consulting shareholders and the public. Training staff. Making tourism responsibility. Undertaking research. (Harding, 2001: 172)

En 1998 el ejemplar 27, aún sin utilizar una terminología específica como la anterior, sí incluye un capítulo introductorio sobre el contexto global de Europa y las tendencias cambiantes del turismo hacia el siglo XXI, esbozando los problemas que puede provocar y el impacto de la legislación europea sobre el sector. 
Son aquellos ejemplares dedicados al turismo en general y con ediciones publicadas tras el año 2000 los que comenzarán a incluir explícitamente y de manera recurrente temas relacionados con el turismo sostenible desde el enfoque medioambiental. Reflejan así las nuevas tendencias sociales y políticas que configuran un nuevo viaje, en busca de algo más que diversión, siendo respetuoso con el enclave natural y con las personas que lo habitan.

En el ejemplar 29 la Unidad 20 utiliza también el término ecoturism, además de actividades relacionadas con la sostenibilidad turística en las unidades 1, 13 y 20.

El 31 también incluye toda una unidad dedicada al turismo sostenible y denominada explícitamente así: Sustainable tourism (Unidad 4). En la unidad 1, además de aportar la propia definición de turismo de la OMT, incluye la de diferentes tipos de turismo, algunas de las cuales ya han sido comentadas previamente en este artículo. Entre ellas destaca en este apartado la del denominado 'Eco-turismo': "For people who want to travel without causing negative impact on the land or the place they visit. Often including activities in close contact with nature and avoiding crowd". (Varela y Bárcena, 2004: 63) Aparece también una terminología más variada, desde el anterior eco-tourism, hasta green tourism, environmental tourism y sustainable tourism. Incluye algo novedoso que no suele aparecer en los demás ejemplares: la crítica ante el exceso de uso de esta terminología para atraer turistas y su falta de contenido en muchas ocasiones; es el llamado Green Washing: "It is a fake type of 'eco-tourism', when some tourist agencies offer Green destinations to atract nature lovers, but then it has nothing to do with it". (Varela y Bárcena, 2004: 63)

El 33 realiza un recorrido por los diferentes tipos de turismo (Old and New Tourism, p. 33) entre los que también aparece Eco-tourism.

En 34, se aborda el tema más profundamente a través de varias unidades completas. La Unidad 6, con Rural Tourism como tema central, incluye Encouraging the customer to be sensitive to the environment (p. 51) o Ecology of The Ecuador (p. 69). La Unidad 10 Specialised Tourism también dedica buena parte a los tipos de turismo sostenible, distinguiendo entre cultural, environmental y rural tourism. En el primero se incluye heritage, tribal, religious, genealogy y research tourism; en el segundo ecotourism, geotourism, nature and wildlife, adventure, alpine, y coastal tourism; el tercer apartado engloba farms/barns, camping, wine/gastronomy, sports, festivals and events y arts and crafts tourism (p. 85). Esta clasificación es tan amplia que denota falta de concreción en la terminología utilizada.

El ejemplar 35 utiliza también el término ecotourism, en la Unidad 2, al tiempo que se refiere a los destination planning and sustainable tourism en la Unidad 9 y a los environmental issues en la Unidad 11. Asimismo, la crítica sobre el exceso en el uso de este tipo de terminología, que aparecía por primera vez en 31 , se incluye de nuevo en la Unidad 8, con el texto Is it as Green as it is painted?

En 36 el autor dedica también un interés considerable a la sostenibilidad medioambiental. La Unidad 7 hace referencia al impacto del turismo en la naturaleza con Global Warming and Tourism y la Unidad 10 Sustainable Tourism se centra exclusivamente en este tema con actividades como ecotourism in your region o putting sustainability to work. 


\subsubsection{Términos más habituales}

La gran cantidad y heterogeneidad de términos utilizados en este apartado hacen necesario un análisis más pormenorizado que en los anteriores. Como hemos visto, su uso no se restringe a los epígrafes principales de unidades o actividades, sino que a menudo subyacen en el contenido de las mismas.

Por orden de uso, el término más utilizado es el de environmental tourism o la alusión a tourism and the environment, que aparece en 13 de los ejemplares analizados que incluyen este parámetro (65\%). También es muy habitual referirse al impacto del turismo como tal con impact of tourism, en 11 ejemplares $(55 \%)$. El siguiente término más comúnmente utilizado es eco-tourism, mencionado en 10 ejemplares (50\%).

Tras ellos, sustainable tourism, seguido de responsible tourism o la expresión how to be a responsible tourist son también comunes, aunque en menor medida (7 y 6 casos, $35 \%$ y $30 \%$, respectivamente). Con una incidencia menor se encuentran los términos social tourism, green tourism y rural tourism. En algunos ejemplares se incluye también geotourism e incluso safe tourism.

\section{Gráfico 6 \\ SOSTENIBILIDAD MEDIOAMBIENTAL. TÉRMINOS MÁS HABITUALES}

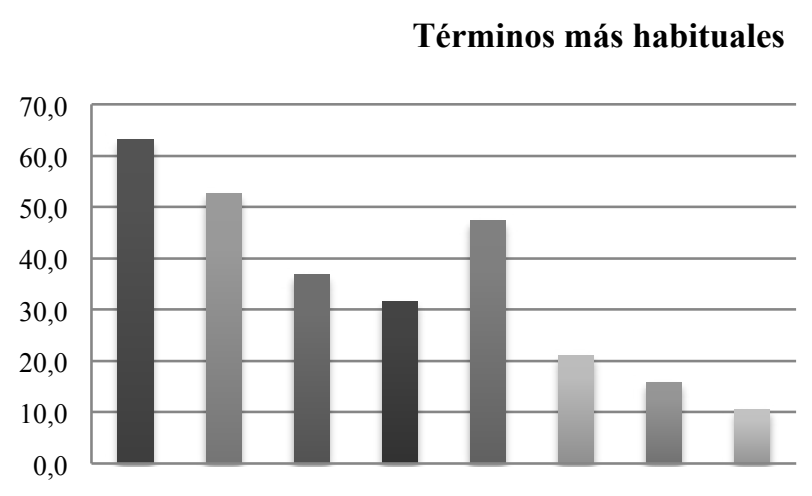

1. Environmental T.

2. The impact of tourism

- 3. Sustainable T.

4. Responsible T.

5. Ecotourism

6. Social T.

7. Green T.

8. Rural T.

La tendencia habitual es la de recurrir a más de un término en el mismo ejemplar, de ahí que la anterior cuantificación muestre el porcentaje de ejemplares en los que aparece cada término, teniendo en cuenta que en la mayoría de los casos estas apariciones se solapan. Esto demuestra también que las diferencias existentes entre las denominaciones no están claramente determinadas pues en muchos casos se utilizan hasta cinco términos diferentes para referirse a aspectos de sostenibilidad medioambiental, como se observa en el siguiente gráfico: 


\section{Gráfico 7 \\ SOSTENIBILIDAD MEDIOAMBIENTAL. \\ NÚMERO DE TÉRMINOS UTILIZADOS EN CADA EJEMPLAR}

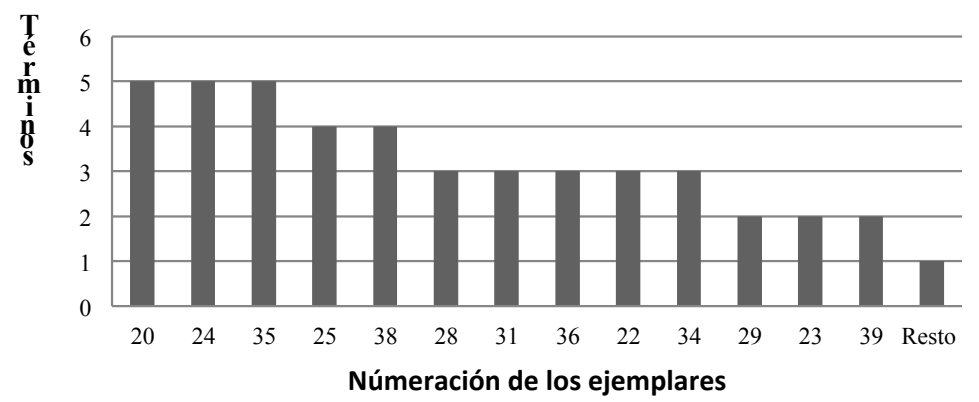

La indefinición existente en la terminología referente a estos temas se observa al detectar que los términos no coinciden en los diferentes ejemplares:

Tres ejemplares utilizan 5 términos diferentes:

20: rural, green, environmental y sustainable tourism junto a the impact of tourism, utilizados de manera indistinta incluso en las mismas unidades o actividades para referirse a la sostenibilidad mediambiental. Sin embargo, este ejemplar es más riguroso que el resto pues incluye definiciones de algunos términos, como rural, green y sustainable tourism. 24: environmental, social, sustainable, responsible y the impact of tourism.

35: ecotourism, environmental, sustainable, responsible y the impact of tourism.

En dos de los ejemplares analizados aparecen 4 términos:

25: environmental, sustainable, responsible y the impact of tourism.

38: green, environmental, sustainable y responsible.

En cinco ejemplares se utilizan 3:

28: environmental, social y the impact of tourism.

31: ecotourism, environmental y sustainable.

36: social, sustainable y responsable.

22: environmental, social y the impact of tourism.

34: rural, environmental y responsible. En este caso sí se distingue entre rural y environmental.

En 3 casos se alude al tema a través de 2 términos:

29: ecotourism y the impact of tourism.

23: ecotourism y environmental tourism.

39: environmental y the impact of tourism.

El resto de ejemplares, salvo en dos casos en los que se alude al concepto sin mencionar ninguno de los términos específicamente acuñados para ello, aparece solamente uno de los términos anteriores de manera indiscriminada, lo que muestra la multiplicidad y falta de consistencia en la terminología empleada. 
Como se observa, los términos más comunes que aparecen en la mayoría de los ejemplares son environmental tourism, sustainable tourism, así como la expresión the impact of tourism.

\subsection{Una visión holística de la sostenibilidad}

La acepción más completa sobre sostenibilidad turística de la OMT comentada al inicio de este artículo, que incluye los cuatro enfoques anteriores, solo aparece en 10 de los 43 ejemplares analizados. 4 de ellos fueron publicados por vez primera en la primera década, los años 90 (20, 22, 23 y 24), 3 durante los primeros cinco años del 2000 (29, 31 y 31) y los 3 restantes corresponden al periodo más cercano a la actualidad, a partir de 2005 (34, 36 y 39). Esto parece indicar que en sus inicios el propio concepto "sostenible" se consideraba más novedoso, por lo tanto menos conocido, y se solía abundar más en sus posibles diferentes acepciones. La tipología de todos estos libros de texto es la de turismo general.

El nivel de competencia lingüística de los ejemplares que incluyen esta visión completa es pre-intermedio en dos casos (23 y 32); de un nivel progresivo que va desde el pre-intermedio al intermedio alto en otros dos (31 y 34); intermedio-alto en cuatro (22, 24,36 y 39) y avanzado en uno (ejemplar 20). El ejemplar 29 no especifica nivel. De aquí parece deducirse que para abordar estos temas el alumnado ya debe haber desarrollado las destrezas lingüísticas en inglés al menos con un nivel intermedio o intermedioalto que le permita entender estos términos específicos, ya que, aunque no se detecta una gradación temporal en la inclusión de temas sobre sostenibilidad que implique un aumento del uso de esta terminología, sí aparece una tendencia de la misma hacia la especialización, como se ha observado en el apartado anterior.

\section{CONCLUSIONES}

Si bien la sostenibilidad es un término actual en boga, sobre el que se basan los proyectos de desarrollo turístico actuales, la distinción de todos los matices englobados en este concepto comenzó a incluirse en los libros de Inglés para Turismo desde la acuñación del propio término en los años 90, cuando la industria turística comienza a concederle más relevancia. En las décadas anteriores estos temas no se solían incorporar en los libros para la enseñanza del inglés turístico, o lo hacían de manera tangencial. Será a partir de la Cumbre Mundial de 1992 cuando comenzaron a aparecer de manera recurrente hasta convertirse en habituales en la mayoría de los ejemplares actuales. Sin embargo, su tratamiento suele restringirse a las dimensiones ambientales y/o culturales de la sostenibilidad establecidas por la OMT. El hecho de que solo 10 de los ejemplares analizados incluyan entre sus contenidos otras dimensiones posibles de la sostenibilidad implica que el concepto holístico aún no está totalmente consolidado en los libros de texto de Inglés aplicado al Turismo y que suele tratarse desde una óptica determinada.

Se ha comprobado cómo los diferentes enfoques en los que se ha categorizado el concepto sostenible se incluyen en los ejemplares en mayor o menor medida dependiendo de su especialidad: la sostenibilidad laboral tiene un papel más relevante en los 
libros de inglés aplicado a la hostelería y restauración; la interculturalidad y las formas saludables de vida se incluyen mayoritariamente en los libros sobre turismo general; las repercusiones medioambientales del turismo son básicas para el sector, incluyéndose habitualmente en los libros sobre turismo general.

En este último caso, la utilización de varios términos solapados para hacer referencia a un mismo concepto pone en evidencia la variedad terminológica existente, que en algunas ocasiones incluso hace necesario acudir a la definición explícita de algunos términos para una correcta comprensión, mientras que en otras esos mismos términos se utilizan como cuasi-sinónimos. Consecuentemente, se constata cómo la propia definición de turismo sostenible en estos ejemplares presenta dificultades, pues la terminología utilizada para referirnos a ese mismo concepto no es consistente.

A partir del año 2000 hay un auge en la publicación libros de texto sobre inglés turístico (Varela, 2007: 335). Aunque no se detecta en ellos una gradación temporal en la inclusión de temas sobre sostenibilidad que implique un aumento y consolidación del uso de una terminología totalmente definida, sí aparece una tendencia de la misma hacia la especialización, enfocando el concepto de sostenibilidad hacia el tema medioambiental principalmente, donde los términos más habituales environmental y sustainable, han pasado a utilizarse de manera recurrente para aludir a la sostenibilidad en los libros de turismo general, como se ha observado en el apartado anterior.

\section{BIBLIOGRAFÍA}

AFZALI, K. y REZAPOORIAN, D. (2014): "Pragmatic aspects of English for Tourism course books and ESL learners' pragmatic needs: A speech act theory perspective". Procedia. Social and Behavioral Sciences, vol. 98, pp. 52-59.

AGRUSA, W., LEMA, J., TANNER, J., HOST, T. y AGRUSA, J. (2010): "Integrating sustainability and Hawaiian culture into the tourism experience of the Hawaiian Islands", Pasos. Revista de Turismo y Patrimonio Cultural, vol. 8, n 2, pp. 247-264.

ALCANTUD, M., RICART, A. y GREGORI, C. (2014): "Share your experience. Digital Storytelling in English for Tourism”, Ibérica, no 27, pp. 185-204.

ALONSO, A. y GONZÁLEZ, L. (2008): "El desarrollo de la competencia intercultural como clave en la adquisición del inglés dentro del contexto específico del turismo", en L. Pérez, I. Pizarro y E. González-Cascos (Coords.), Estudios de metodología de la lengua inglesa (IV). Valladolid, Universidad de Valladolid, pp. 187-200.

AREIZAGA, E., GÓMEZ, I. e IBARRA, E. (2005): "El componente cultural en la enseñanza de lenguas como línea de investigación”, Revista de Psicodidáctica, Vol. 10, $\mathrm{n}^{\circ} 2$, pp. 27-46.

BÁRCENA, E., MARTÍN, E. y JORDANO, M. (2016): "Innovación metodológica y tecnológica en la enseñanza del inglés para turismo a distancia", Ibérica, n 31, pp. 39-62.

BOCANEGRA, A. (2015): "La competencia intercultural en los manuales de texto con fines específicos a través de la evaluación de sus indicadores y niveles de dominio", Cuadernos de Filología Francesa, vol. 26, pp. 29-43.

CABELLOS, M.R. y CASTILLO G.S. (2002): "Un estudio comparativo de distintos métodos de inglés para turismo", en L. Sierra y E. Fernández (Coords.), Lenguas para 
fines específicos VII. Investigación y Enseñanza. Alcalá de Henares, Universidad de Alcalá de Henares, pp. 207-216.

CALVI, V. (2010): "Los géneros discursivos en la lengua del turismo: una propuesta de clasificación”, Ibérica, n 19 , pp. 9-32.

COMISIÓN DE LAS COMUNIDADES EUROPEAS (CCE) (1990): Medidas comunitarias para el fomento del turismo rural. COM, 438, Bruselas.

CUESTAS, A., ZAMUNER A. y PERALTA, S. (2013): “Un permanente desafío en el ámbito del inglés con fines específicos: El desarrollo de la competencia comunicativa intercultural", Puertas abiertas: Revista de la Escuela de Lenguas, 9. Disponible en: www.puertasabiertas.fahce.unlp.edu.ar/numeros/numero-9/

GONZÁLEZ, D.M. (2012): Análisis descriptivo de la traducción de culturemas en el texto turístico. Tesis Doctoral inédita. Universidad Politécnica de Valencia.

GONZÁLEZ, G., FRÍAS, R. y GÓMEZ, O. (2014): "Proposal of a procedure to analyze the faults in a service of catering lodge", Rotur: Revista de Ocio y Turismo, no 7, pp. 20-40.

KUPPAN, A. (2008): An Evaluation of an English Course for Hospitality Management in a Tertiary Institution. Kuala Lumpur, University of Malaya. Disponible en: http://repository.um.edu.my/679/1/Ajuntha\%20Kuppan.pdf

LANE, B. (2011). "Sustainable rural tourism strategies: A tool for development and conservation". Revista Interamericana de Ambiente y Turismo-RIAT, vol. 1, n 1, pp. 12-18.

LARIO, M.C. y VÁZQUEZ, M. (2013): “The intercultural component in Business English textbooks", Iberica, no 26, pp. 171-194.

LOERA, E. y ESCALERA, A. (2014): "El inglés como factor detonador en la operación turística”, en T. J. Cuevas y A. Palafox (Coords.), Complejidad del turismo en la frontera del conocimiento. Ciudad Juárez, Gasca, pp. 169-185.

MOREIRA, P., LOPES, L. y DE ABREU, M. (2015): "El turismo como núcleo de estudio interdisciplinario. [Re]construcción de los procedimientos y adecuaciones metodológicas". Estudios y Perspectivas en Turismo, vol. 24, n 3, pp. 450-469.

OLIVEIRA, I., ROBERTO, T. y COSTA, C. (2014): “Tourism English Programmes: Do they mirrow the reality and the contents of the tourism industry?", Revista Turismo $y$ Desenvolvimiento, Vol. 1, n 21-22, pp. 299-313.

ORGANIZACIÓN MUNDIAL DE TURISMO (OMT) (1991): Conferencia Internacional de Estadísticas de Turismo y Viajes, Ottawa, OMT.

ORGANIZACIÓN MUNDIAL DE TURISMO (OMT) (1998): Introducción al Turismo. Madrid, ORGANIZACIÓN MUNDIAL DE TURISMO (OMT) (1999): Agenda para planificadores locales: turismo sostenible y gestión municipal. Madrid, OMT.

ORGANIZACIÓN MUNDIAL DE TURISMO (OMT) (2012): Turismo y sostenibilidad. Disponible enhttp://cf.cdn.unwto.org/sites/all/files /docpdf/turismoysostenibilidad_0.pdf ORGANIZACIÓN MUNDIAL DE TURISMO (OMT) (2015): Panorama OMT del Turismo Internacional. Disponible en http://www.e-unwto.org/doi/pdf/10.18111/9789284416875 ORGANIZACIÓN MUNDIAL DE TURISMO (OMT) (2017a): El turismo internacional mantiene un crecimiento sostenido pese a las dificultades. Comunicado de prensa 18/01/17. Disponible en http://www2.unwto.org/es/press-release/2017-01-17/el-turismointernacional-mantiene-un-crecimiento-sostenido-pese-las-dificul 
ORGANIZACIÓN MUNDIAL DE TURISMO (OMT) (2017b): 2017 Año Internacional del Turismo Sostenible para el Desarrollo. Disponible en http://www2.unwto.org/es/ tourism4development2017

ORGANIZACIÓN DE NACIONES UNIDAS (1987): Nuestro futuro común. Informe Brundtland. Disponible en www.un.org/es/comun/docs/?symbol=A/42/427.

PÉREZ, L.P. (2010): "Reflexiones en torno al enoturismo y el turismo rural: análisis de necesidades en la oferta a clientes de habla inglesa en Castilla y León”, en M.T. Ramos (Coord.), En torno al vino: estudios pluridisciplinares. Valladolid, Universidad de Valladolid.

PÉREZ, M. (2004): Manual del turismo sostenible: cómo conseguir un turismo social, económico y ambientalmente responsable. Madrid, Mundiprensa.

PÉREZ, M., PUPO, S. y BUENAVENTURA, A. (2011): "Metodología para la elaboración de libros de texto en la enseñanza de inglés con fines turísticos". Ciencias Holguin, vol. 17, $\mathrm{n}^{\mathrm{o}} 2$, pp. 1-9.

PICCIONI, S. (2014): “Cortesía y lenguas de especialidad entre lo local y lo global: el caso de las reseñas de hoteles en español e inglés", Normas, n 4, pp. 93-116.

PRÁDANOS, L. (2015): "La enseñanza del español en la era del antropoceno: Hacia la integración de la sostenibilidad en las clases de español como lengua extranjera", Hispania, vol. 98, n 2, pp. 333-345.

REYNOLDS, P. (2013): "Hotel companies and corporate environmentalism", Tourism \& Management Studies, vol. 9, n 1, pp. 7-12.

RÍO, C. (2012): "Hacia una caracterización del inglés para el turismo en el ámbito universitario", en K. Rohr and I. K. León (Coords.), Unternehmen und Kommunikation: Festschrift für Carmen Reyero Hernández. San Cristóbal de La Laguna, Universidad de La Laguna, pp. 275-288.

RODRÍGUEZ, J. (2013): Las Guías de Buenas Prácticas como Instrumento para el Desarrollo de un Turismo Sostenible: El Caso de Sierra de las Nieves. Tesis Doctoral inédita. Universidad de Cádiz.

SOTO, J. (2013): "La traducción de términos culturales en el contexto turístico españolinglés: recepción real en usuarios anglófonos", Quaderns. Revista de Traducció, $\mathrm{n}^{\mathrm{o}}$ 20, pp. 235-250.

SUAU, F. (2016): "What can the discursive construction of stance and engagement voices in traveller forums and tourism promotional websites bring to a cultural, cross-generic and disciplinary view of interpersonality?", Ibérica, n 16, pp. 199-220.

VARELA, R. (2007): "Hacia una caracterización del inglés para fines específicos (Turismo)", Didáctica (Lengua y Literatura), vol. 19, pp. 327-345.

VARÓN, M.E. (2009): “Componente cultural, libros de texto y enseñanza del inglés como lengua extranjera”, Forma y función, vol. 22, n 1, pp. 95-124.

WEAVER, D.B. (2006): Sustainable tourism: Theory and practice. Londres, Routledge. VIDAL, K., BERLANGA DE JESÚS, L. y RUMOLD, G. (2009): "Los nuevos retos del conocimiento de idiomas: inglés, francés, alemán”, en J. M. Rodríguez y M. M. Alonso (Coords.), Nuevas tendencias y retos en el sector turismo: un enfoque multidisciplinar. Madrid, Delta, pp. 313-331. 


\section{ANEXO. MANUALES ANALIZADOS (ORDEN CRONOLÓGICO DE PUBLI- CACIÓN)}

\subsection{Hostelería y restauración}

[1] REVELL, R. y STOTT, C. (1982) (1994): Five Star English for the Hotel and Tourist Industry. Oxford, O.U.P.

[2] YATES. J. (1990): May I help you. Harlow, Prentice Hall.

[3] HARDING, K. y HENDERSON, P. (1994) (2000). High Season (English for the Hotel and Tourist industry. Oxford, O.U.P.

[4] STOTT, T. y BUCKINGHAM, A. (1995) (2003): At your service (English for the Tourist Industry). Oxford, O.U.P.

[5] POHL, A. (1996) (2004): Test your Professional English for Hotel and Catering. Londres, Penguin.

[6] FITZGERALD, H. (1998) (2002): Cross Cultural Communication for the Tourism and Hospitality Industry. Melbourne, Pearson.

[7] TALALLA, R. (2000) (2008): English for Restaurant Workers. Compass.

[8] O'HARA, F. (2002): Be my guest (English for the Hotel Industry). Cambridge, C.U.P.

[9] BAUDE, A., IGLESIAS, M. y IÑESTA, A. (2002) (2004): Ready to Order. Essex, Longman.

[10] THOMAS, J. (2003) (2004): English for Hospitality and Food Service. Harlow, Prentice Hall.

[11] STOTT, T. y REVELL, R. (2004) (2008): Highly Recommended 1 (English for the Hotel and Catering Industry). Oxford, O.U.P.

[12] STOTT, T. y POHL, A. (2004) (2010): Highly Recommended 2 (English for the Hotel and Catering Industry). Oxford, O.U.P.

[13] ZWIER, L.J. y CAPLAN, N. (2007): Everyday English for Hospitality Professionals. Compass.

[14] HEPBURN, E. y MCALLISTER, J. (2008a): Hospitality (Intermediate 1). Edimburgo, Leckie and Leckie.

[15] HEPBURN, E. y MCALLISTER, J. (2008b): Hospitality (Intermediate 2). Edimburgo, Leckie and Leckie.

[16] SEYMUR, M. (2011). Hotel and Hospitality English. Londres, Collins.

[17] EVANS, V., DOOLEY J. y GARZA, V. (2011): Hotels and Catering (Career Paths). Newbury, Express Publishing.

\subsection{Turismo general}

[18] STOTT, T. y HOLT, R. (1991) (2003): First Class (English for Tourism). Oxford, O.U.P.

[19] GALlEGO, J. MIODUSZEWSKA, M., OLIVARES, A. y URQUÍA, M.A. (1992): Checkpoint (English in Tourism). Madrid, Ramón Areces.

[20] MIODUSZEWSKA, M., GALLEGO, J., OLIVARES, A. y URQUÍA, M.A. (1992) (1994): Checkpoint 2 (English in Tourism). Madrid, Ramón Areces.

[21] WYATT, R. (1995) (2007): Check your English Vocabulary for Leisure, Travel and Tourism. Londres, AyC Black. 
[22] COOK, R.A., YALE, L.J. y MARQUA, J.J. (1995) (2010): Tourism. The Business of Travel. Harlow, Prentice Hall.

[23] DUBICKA, I., O'KEEFFE, M. y STRUTT, P. (1997) (2005): English for International Tourism 1. Essex, Longman.

[24] DUBICKA, I., O'KEEFFE, M. y STRUTT, P. (1997) (2013): English for International Tourism 3. Essex, Longman.

[25] HARDING, K. (1998) (2001): Going International (English for Tourism). Oxford, O.U.P. [26] JONES, L. (1998) (2002): Welcome. Cambridge, Cambridge University Press.

[27] DAVIDSON, R., (1998): Travel and Tourism in Europe. Essex, Longman.

[28] ROWE, A., SMITH J.D. y BOREIGN, F. (2002): Travel and Tourism. Standard level. Cambridge, C.U.P.

[29] WOOD, N., (2003): Tourism and Catering. Oxford, O.U.P.

[30] USÓ, E., FORTANET I. y SAORÍN, A.M. (2004): Starting English for Tourism Studies. Castellón, Universidad Jaime I.

[31] VARELA, R. y BÁRCENA, E. (2004): English in the Tourist Industry. Madrid, Ramón Areces.

[32] TAYLOR, L. (2004) (2008): International Express. Oxford, O.U.P.

[33] WALKER, R. y HARDING, K. (2006) (2011a): Tourism 1 (Provision). Oxford, Oxford English for Careers.

[34] WALKER, R. y HARDING, K. (2007) (2011b): Tourism 2 (Encounters). Oxford, Oxford English for Careers.

[35] MOL, H. (2008) (2009): English for Tourism and Hospitality. Reading, Garnet Education.

[36] WALKER, R. y HARDING, K. (2009): Tourism 3 (Management). Oxford, Oxford English for Careers.

[37] EVANS, V., DOOLEY J. y GARZA, V. (2011) (2012): Tourism (Career Paths). Newbury, Express Publishing.

[38] DUBICKA, I., O'KEEFFE, M. y STRUTT, P. (2013): English for International Tourism 2. Essex, Longman.

[39] COOK, R.A., HSU, C. J.y MARQUA, J.J. (2014): Tourism. The Business of Hospitality and Travel. Harlow, Prentice Hall.

\subsection{Viajes aéreos}

[40] AKIYAMA, C. (1976): The Language of Air Travel in English. In flight services. Harlow, Prentice Hall.

[41] EUGENE, J. (1976): The language of Air Travel in English. Ground Services. Harlow, Prentice Hall.

[42] GERIGHTY, T. y DAVIS, S. (2001): English for Cabin Crew. Hampshire, Cengale Learning.

\subsection{Oficina de turismo}

[43] ESHOO, P. (2005): May I help you? Training Manual for Tourist Information. Mekomg, Mekomg Tourism Development Project. 